\title{
Liposarcoma paratesticular gigante
}

\section{Giant Paratesticular Liposarcoma}

\author{
Carlos Cortes-Caballero ${ }^{1}$ Margarita Rosa Mogollon-Zehr ${ }^{2}$ Silvia Nathalia Vera Campos ${ }^{2}$ \\ ${ }^{1}$ Universidad de Antioquia, Patólogo egresado de The Western \\ Pennsylvania Hospital, Pittsburg, Pennsylvania, Estados Unidos de \\ América \\ ${ }^{2}$ Curso de Medicina, Universidad Industrial de Santander, Santander, \\ Colombia \\ Address for correspondence Carlos Cortes Caballero, MD, Médico
Cirujano, Bucaramanga, Colombia (e-mail: cccaballero@gmail.com).

\section{Resumen}

Palabras Clave

- liposarcoma paratesticular

- liposarcoma

- sarcoma

- tumor paratesticular

- cordón espermático

- hernia inguinal
Introducción Los liposarcomas son lesiones que se originan en el tejido mesodérmico, su localización paratesticular es muy rara. Se divide en 4 subtipos histológicos, siendo el mixoide el más infrecuente. Tiene una recurrencia del $70 \%$, Por lo que su tratamiento inicial debe ser agresivo.

Objetivo resaltar su gran tamaño y que sea considerado como diagnóstico diferencial de masa inguinoescrotal.

Presentación del caso Paciente masculino de 87 años con masa dolorosa inguinoescrotal derecha con diagnóstico de hernia inguinal unilateral, se realiza orquiectomía inguinal derecha. En patología se recibe pieza quirúrgica de $2440 \mathrm{~g}$; al estudio histológico se observa lesión tumoral mesenquimal maligna, rodeado por estroma nodular mixoide prominente. Se diagnostica liposarcoma mixoide de cordón espermático. (Figura 2).

Conclusiones Son neoplasias raras mal diagnosticadas como hernia inguinal, escrotal o lipoma de la médula espermática. Tiene un alto riesgo de metástasis, siendo la orquiectomía inguinal con resección del cordón espermático el manejo quirúrgico ideal.

Introduction Liposarcomas are lesions originated in the mesodermal tissue, paratesticular location is very rare. It is divided into 4 histological subtypes, being the most uncommon the myxoid. It has a recurrence of $70 \%$, so the treatment should be aggressive.

Objective to emphasize the large size and the differential diagnosis of inguinoescrotal mass.

Case Presentation 87-year-old male patient with right inguinoescrotal pain with diagnosis of unilateral inguinal hernia, right inguinal orchiectomy is performed. A surgical specimen of $2440 \mathrm{~g}$ is received in pathology. Histological study shows malignant mesenchymal tumor lesion, surrounded by prominent myxoid nodular stroma. Myxoid liposarcoma of the spermatic cord is diagnosed. (Fig. 2).

Conclusions they are rare neoplasms and are poorly diagnosed as inguinal hernia, scrotal or lipoma of the spermal cord. It has a high risk of metastasis, the ideal surgical management is an inguinal orchiectomy with resection of the spermatic cord. received

September 18, 2018

accepted

September 28, 2018
DOI https://doi.org/

$10.1055 / \mathrm{s}-0038-1675424$.

ISSN 0120-789X.

e ISSN 2027-0119.
Copyright (c) 2019, Sociedad Colombiana License terms de Urología. Publicado por Thieme Revinter Publicações Ltda., Rio de Janeiro, Brazil. Todos los derechos reservados. 


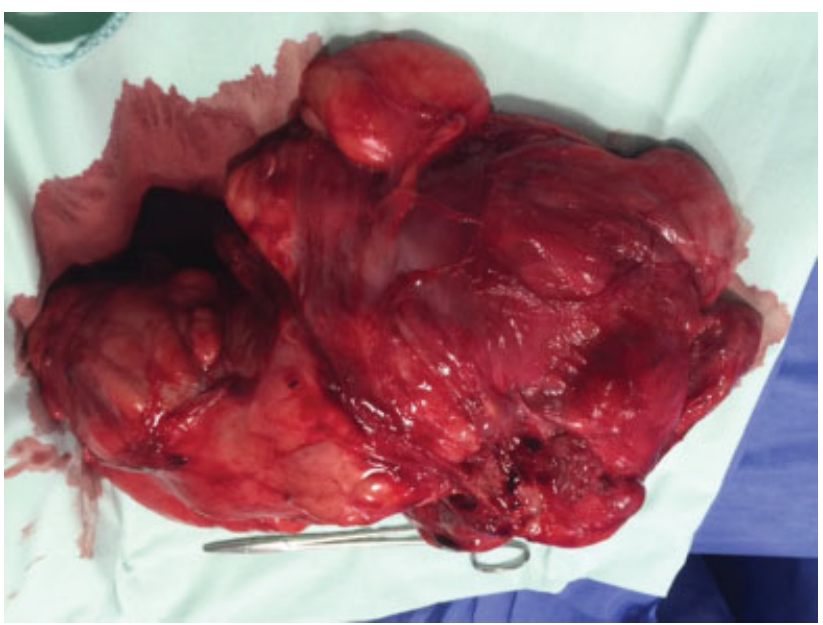

Fig. 1 Pieza quirúrgica de aspecto externo.

\section{Introducción}

Los liposarcomas son neoplasias que pueden desarrollarse en cualquier sitio donde exista tejido adiposo, ya que se originan a partir del mesénquima, su localización principal es el retroperitoneo; los tumores primarios paratesticulares son muy raros con una incidencia de 0,3 casos en un millón de habitantes, que corresponden al $20 \%$ de los sarcomas ${ }^{1}$. Su origen se encuentra en: el cordón espermático (76\%), las túnicas testiculares (20\%) y el epidídimo $(4 \%){ }^{2}$ El primer caso notificado de liposarcoma paratesticular fue descrito por Lesauvage en $1845 .^{3}$

La orquiectomía radical con una amplia escisión local es el método terapéutico ideal, su diagnóstico prequirúrgico es muy poco frecuente, sin embargo, cuentan con un buen pronóstico y un alto índice de sobrevida a 5 años. ${ }^{4}$

Se presenta un caso de un liposarcoma mixoide originado en cordón espermático, con el objetivo de resaltar su gran tamaño y que sea uno de los diagnósticos diferenciales considerados ante la presencia de masa inguinoescrotal.

\section{Caso Clínico}

Paciente masculino de 87 años de edad. Acude por cuadro clínico de 3 años de evolución caracterizado por la presencia de una masa inguinoescrotal derecha que aumentó progresivamente de tamaño, acompañado por sensación de pesadez y dolor; al examen físico se encuentra masa inguinal derecha, fija, de bordes irregulares, que no presenta translucencia y la cual por su tamaño no es posible verificar si es reducible. Por la clínica se realiza diagnóstico de hernia inguinal unilateral derecha, para la confirmación del diagnóstico se realiza estudio de ultrasonido que reporta masa paratesticular sólida y heterogénea, pero no se puede descartar compromiso testicular, por lo cual se ordena orquiectomia inguinal derecha, se extrae pieza para patología (-Fig. 1).

En el laboratorio se recibe pieza quirúrgica de $2440 \mathrm{~g} \mathrm{y}$ con dimensiones de $25 \times 20 \mathrm{~cm}$. En la superficie externa se observan múltiples adherencias de color amarillo. A los cortes seriados se identifican lesiones nodulares, la mayor de ellas mide $15 \times 9 \mathrm{~cm}$, el tejido es homogéneo y de consistencia blanda (-Fig. 2).

Al estudio microscópico se aprecia una lesión tumoral mesenquimal maligna compuesta por células lipogénicas, redondas, ovales y lipoblastos pequeños, rodeado por un estroma nodular mixoide prominente, acompañado por una vasculatura delicada y ramificada que infiltra de forma difusa. El tejido testicular y el epidídimo no presentan alteraciones. Se hace diagnostico microscópico de liposarcoma mixoide del cordón espermático (-Fig. 3).

\section{Discusión}

Los liposarcomas son neoplasias malignas originadas del tejido mesenquimal y se dividen en cuatro subtipos histológicos: células bien diferencias, mixoides o redondas, pleomórficas y desdiferenciadas. ${ }^{5}$

Son neoplasias de crecimiento lento, por lo general con una evolución mayor a 10 años, su tamaño varía entre 1,5 y $23 \mathrm{~cm}$. La edad de presentación más común está entre los 50-

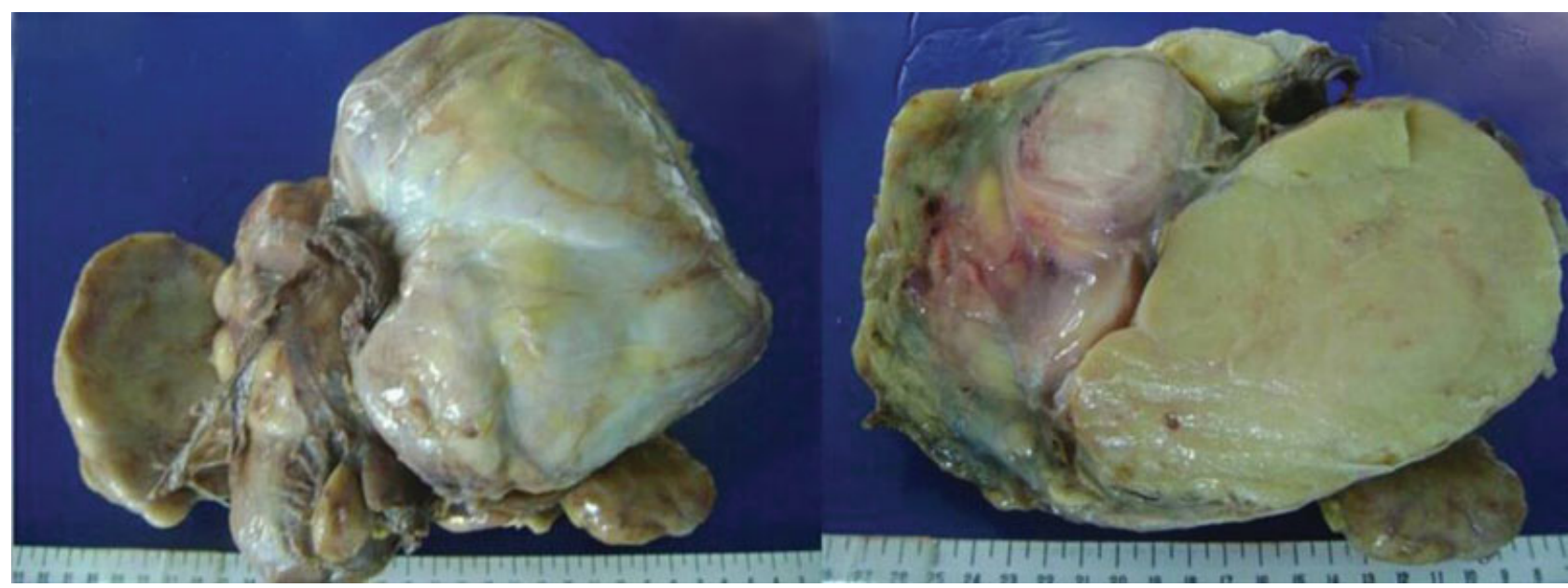

Fig. 2 Pieza quirúrgica de superficie externa e interna. 


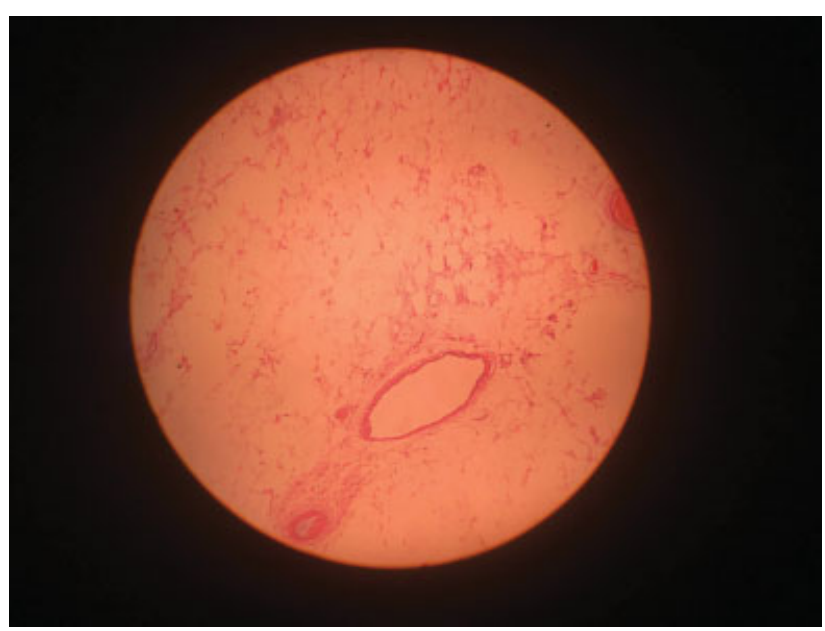

Fig. 3 Imagen histológica en la que se observa células adiposas.

60 años. ${ }^{4}$ Aunque es una entidad con mayor frecuencia en la edad adulta, se reporta un caso de paciente de 87 años que no corresponde a la edad promedio de presentación, al igual que su tiempo de evolución menor a 10 años.

Los liposarcomas paratesticulares suelen tener un difícil diagnóstico, debido a su poca frecuencia de presentación, para eso se pueden realizar estudios imagenológicos que ayuden a definir los márgenes del tumor, las características del tejido, el compromiso linfático, el estadio y la presencia de metástasis a órganos viscerales; eso acompañado de una clínica de presentación de masa solida con crecimiento lento, no translucente, no reducible e indolora. ${ }^{6}$ En nuestro caso, se hizo un diagnóstico erróneo de hernia inguinal debido a la clínica inusual del paciente, por lo tanto, es importante mencionar entre los diagnostico diferenciales clínicos del liposarcoma paratesticular la hernia inguinal, hidroceles, lipomas y angiomiolipomas. ${ }^{7,8}$

Es preciso realizar la diferenciación con sus subtipos histológicos ya que suelen ser confundidos con los liposarcomas bien diferenciados, que en ocasiones contienen estroma mixoide. Por otro lado, el liposarcoma paratesticular puede resultar difícil de diferenciar de los lipomas, los cuales corresponden a la mayoría de tumores adiposos del cordón espermático; sin embargo, los liposarcomas suelen ser de mayor tamaño. ${ }^{3}$ En nuestro caso, se halló la presencia de una lesión de crecimiento rápido y gran tamaño, acompañada de sintomatología, características sugestivas de malignidad y de mal pronóstico, contrario a lo reportado en la literatura.

El ultrasonido es el método diagnóstico más utilizado para masas escrotales, tiene una sensibilidad de $95-100 \%$ para distinguir lesiones extratesticulares de las intratesticulares., ${ }^{5,9}$ En esa patología es frecuente encontrar una lesión sólida, hiperecoica y heterogénea, lo cual coincidió con los hallazgos encontrados en este caso. Sin embargo, el diagnóstico definitivo se hace mediante el estudio histopatológico.

El tratamiento ideal es la orquiectomía radical con escisión en bloque, acompañado de un seguimiento a largo plazo debido a su alta tasa de recurrencia. La escisión completa del tumor es una dificultad que suele presentarse debido a su ubicación anatómica, el tamaño del tumor y la infiltración de tejidos adyacentes, ${ }^{10}$ por lo cual se recomienda la asociación del abordaje quirúrgico con la radioterapia y quimioterapia. Pese a eso, la quimioterapia sigue siendo controversial y la radioterapia es considerada principalmente para el manejo de recurrencias. En nuestro caso debido al volumen de la masa y la persistencia del dolor, se realizó intervención quirúrgica, por sus posibles complicaciones.

La recurrencia local puede aparecer en el escroto, área inguinal, pelvis o sus zonas vecinas, siguiendo la dirección de los conductos deferentes. La recurrencia de los liposarcomas es alta (50-70\%) y por lo general tardía; la principal localización es a nivel intraescrotal (30\%). Los factores que aumentan las recurrencias son: la localización inguinal, manipulación previa del tumor, grado de diferenciación y la profundidad de invasión. ${ }^{2}$ En relación a nuestro caso, el tamaño no parece tener un efecto significativo en cuanto a la recurrencia del tumor, sin embargo, sí que es un factor a considerar de acuerdo a su localización y subtipo histológico.

\section{Conflicto de intereses}

Los autores declaran no tener ningún conflicto de intereses.

\section{Referencias}

1 Sopeña-Sutil R, Silan F, Butron-Vila MT, Guerrero-Ramos F, LagaronComba E, Passas-Martinez J. Multidisciplinary approach to giant paratesticular liposarcoma. Can Urol Assoc J 2016;10(9-10): E316-E319 Doi: 10.5489/cuaj.3346

2 Müller Arteaga C, Egea Camacho J, Álvarez Gago T, Cortiñas González JR, Gonzalo Rodríguez V, Fernández del Busto E. Liposarcoma de cordón espermático. Asociación con carcinoma de próstata. Comunicación de un caso y revisión de la literatura. Actas Urol Esp 2005;29(07):700-703

3 Tajima S, Koda K. Paratesticular dedifferentiated liposarcoma with prominent myxoid stroma: report of a case and review of the literature. Med Mol Morphol 2017;50(02):112-116

4 García Morúa A, Lozano Salinas JF, Valdés Sepúlveda F, Zapata H, Gómez Guerra LS. Liposarcoma del cordón espermático: nuestra experiencia y revisión de la literatura. Actas Urol Esp 2009;33 (07):811-815

5 Pănuş A, Meşină C, Pleşea IE, et al. Paratesticular liposarcoma of the spermatic cord: a case report and review of the literature. Rom J Morphol Embryol 2015;56(03):1153-1157

6 Gómez Dorronsoro ML, Pascual Piédrola I, Córdoba IturriagaGoitia A, Valenti Ponsa C, Manrique Celada M, Garrón Aoiz L. Liposarcoma de cordón espermático: criterios de diagnóstico diferencial y tratamiento. Arch Esp Urol 2000;53(01):65-67

7 Murad V, Aponte WO, Romero JA. Angiomiolipoma renal: nuevas perspectivas. Revista Urología Colombiana 2016;XXV(01):16-24

8 Rivero WR, Pradilla JE, Orozco M. Angiomiolipoma renal gigante y linfangioleiomiomatosis pulmonar esporádica no filiada. A propósito de un caso. Revista Urología Colombiana 2014;XXIII (03):227-232

9 Ávila-Boza MLP, Ángeles-Fernández JC, Aguirre-Quezada DE. Liposarcoma desdiferenciado del cordón espermático. Rev Mex Urol 2012;72(06):307-309

10 Bachmann R, Rolinger J, Girotti P, et al. Liposarcoma of the spermatic cord: impact of final surgical intervention-An institutional experience. Int J Surg Oncol 2016;2016:4785394 